I. 


\title{
Krise der Kritik \\ Zur Poetik von Kasus und Rätsel am Beispiel zweier Kriminalerzählungen von Jodokus D.H. Temme und Auguste Groner
}

\begin{abstract}
Die Literaturwissenschaft hat lange gebraucht, bis sie sich darauf einigen konnte, dass es sich bei der Kriminalliteratur tatsächlich um Literatur handelt. ${ }^{1}$ Danach hat sie sich noch einmal so lange mit der Frage herumgeschlagen, wie sich die Kriminalliteratur in die etablierte Gattungslandschaft einfügt: Ist Kriminalliteratur lediglich eine Untergattung der Trivialliteratur? ${ }^{2}$ Wie verhalten sich Kriminalerzählung und Detektiverzählung zueinander? ${ }^{3}$ Wie Verbrechens- und Kriminalliteratur? ${ }^{4}$ Gibt es bestimmende Gattungs-
\end{abstract}

1 So schrieb etwa der Literaturhistoriker Adolf Stern, offenbar auch mit Blick auf seinen Zeitgenossen Jodokus D.H. Temme, dass es sich bei der Kriminalliteratur um "die gefährlichste und widrigste Specialität der Tagesbelletristik « handle, und er sorgte sich um »die Grenze zwischen der berechtigten poetischen Darstellung und der unberechtigten Abart" (Adolf Stern: Der Kriminalroman und die Kriminalnovelle [1864], in: Realismus und Gründerzeit. Manifeste und Dokumente zur deutschen Literatur 1848-1880, hg. von Max Bucher u.a., Stuttgart 1975, Bd. 2, S. 301-304, hier S. 301).

2 Dieser These widerspricht etwa Gerhard Schmidt-Henkel: Kriminalroman und Trivialliteratur, in: Der wohltemperierte Mord. Zur Theorie und Geschichte des Detektivromans, hg. von Viktor Žmegač, Frankfurt a.M. 1971, S. 149-176.

3 Helmut Heißenbüttel hat den Kriminalroman kurzerhand mit dem Detektivroman identifiziert (Helmut Heißenbüttel: Spielregeln des Kriminalromans [1963/1966], in: Der Kriminalroman. Poetik - Theorie - Geschichte, hg. von Jochen Vogt, München 1998, S. 111120, hier S. 113). Ihm widerspricht Hans-Otto Hügel: Untersuchungsrichter, Diebsfänger, Detektive. Theorie und Geschichte der deutschen Detektiverzählung im 19. Jahrhundert, Stuttgart 1978, S. 19f.

4 Richard Gerber etwa will die Kriminalliteratur der Verbrechensliteratur unterordnen (Richard Gerber: Verbrechensdichtung und Kriminalroman [1966], in: Der Kriminalroman, S. 73-83). Peter Nusser spricht sich hingegen für eine Nebenordnung und damit für eine Einordnung auf gleicher gattungshierarchischer Stufe aus. Deswegen verteidigt er bis in die jüngste Auflage seines Standardwerks zum Kriminalroman die Unterscheidung zwischen Verbrechensliteratur, die den Verbrecher psycho- und soziologisiert, und Kriminalliteratur, die das Aufdecken des Verbrechens in den Vordergrund stellt (Peter Nusser: Der Kriminalroman, 4., akt. u. erw. Aufl., Stuttgart 2009, S. VI, 1f.). Diese Eingrenzung des Untersuchungsbereiches wurde von Rezensenten wiederholt kritisiert (Peter Stoll: Der Kriminalroman [...], in: Informationsmittel (IFB). Digitales Rezensionsorgan für Bibliothek und Wissenschaft 12 (2004), http://swbplus.bsz-bw.de/bsz105819069rez.pdf [Stand: 11.11.2014]; Thomas Wörtche: Ein Konzept stößt an seine Grenzen, in: IASLonline. http://www.iaslonline.de/index.php?vorgang_id=3159 [Stand: 11.11.2014]). 
merkmale? ${ }^{5}$ Oder lässt sich der Kriminalroman vielleicht überhaupt nicht abgrenzen, weil in fast jedem Roman ein Verbrechen stattfindet? ${ }^{6}$

Wenn man bedenkt, zu welch beckmesserischen Auseinandersetzungen diese Gattungsdiskussionen bisweilen geführt haben - etwa bei der müßigen Suche nach einer sersten Detektiverzählung $^{7}-$, dann kann man einschätzen, wie wichtig die kulturwissenschaftliche Wende für die wissenschaftliche Beschäftigung mit Kriminalliteratur war. Die US-amerikanische law-and-literature-Forschung ${ }^{8}$ und ihre deutsche Variante im Feld $>$ Recht und Literatur ${ }^{9}$ haben gezeigt, dass die Texte erst in einem juristischen und kriminalistischen Diskursfeld ihre Bedeutung entfalten.

Dieser Beitrag stellt den Versuch dar, den älteren gattungstheoretischen Ansatz mit einer kulturwissenschaftlichen Perspektive zu verbinden. Der Motivzusammenhang von Tötungsarten und Ermittlungspraxen soll exemplarisch verdeutlichen, dass die unterschiedlichen poetischen Formen der Kriminalliteratur pragmatische Funktionen erfüllen, genauer gesagt, dass die Kriminalliteratur ein Medium ist, in dem das abstrakte Spannungsfeld von kollektiver Rechtsnorm und individuellem Rechtsbruch auf spezifisch literarische Weise beschreibbar und problematisierbar wird. Die Grundannahme dieses Beitrags lautet also, dass die Kriminalliteratur mit den ihr eigenen Mitteln das zentrale politische Problem bearbeitet, wie eine gute Sozialordnung gestaltet werden kann.

5 Nach solchen Merkmalen suchen vor allem (aber beileibe nicht nur) strukturalistisch geprägte Gattungstypologien. Als Beispiel sei nur genannt Tzvetan Todorov: Typologie des Kriminalromans [1966], in: Der Kriminalroman, S. 208-215. Einen guten Überblick zur Merkmalsdebatte bietet Peter Nusser: Der Kriminalroman, S. 23-68.

6 So argumentiert Richard Alewyn: Anatomie des Detektivromans [1968/1971], in: Der Kriminalroman, S. 52-72, hier S. 52f.

7 Auslöser der Diskussion war der wichtige Beitrag von Richard Alewyn: Die Anfänge des Detektivromans, in: Der wohltemperierte Mord. Zur Theorie und Geschichte des Detektivromans, hg. von Viktor Žmegač, Frankfurt a.M. 1971, S. 185-202, hier S. 194-202. Für einen Überblick dieser Diskussion vgl. Detlef Kremer: E.T.A. Hoffmann. Erzählungen und Romane, Berlin 1999, S. 147f.

8 Eine gute Zusammenfassung dieser Debatte bietet Ian Ward: Law and Literature. A Continuing Debate, in: ders.: Law and Literature. Possibilities and Perspectives, Cambridge 1995, S. 3-27.

9 Vgl. Bernhard Greiner: Das Forschungsfeld `Recht und Literatur, in: Recht und Literatur. Interdisziplinäre Bezüge, hg. von Bernhard Greiner, Barbara Thums und Wolfgang Graf Vitzthum, Heidelberg 2010, S. 7-26. Einen Eindruck über die Produktivität der amerikanischen und deutschen Forschung vermittelt Thomas Sprecher: Literatur und Recht. Eine Bibliographie für Leser, Frankfurt a.M. 2011 - insbesondere wenn man bedenkt, dass selbst diese umfangreiche Bibliographie nur eine Auswahl verzeichnen kann, in der beispielsweise Einträge zu Groner und Temme fehlen. 
Das vorausgesetzt, wird in historischer Perspektive das ausgehende 19. Jahrhundert zu einem besonders interessanten Untersuchungszeitraum, weil sich zu dieser Zeit ein Umbruch in der Gattungsgeschichte der Kriminalliteratur ereignet, ${ }^{10}$ der die Frage nach seinen sozialen Ursachen aufwirft. Von der Spätaufklärung bis an die Schwelle zur frühen Moderne folgt die Kriminalliteratur typischerweise einer Poetik des Kasus: Sie erzählt einen Einzelfall, um am Beispiel dieses Falles abstrakte Normen an irgendeinem übergeordneten Wert zu messen und so die Differenz zwischen Legalität und Legitimität sichtbar zu machen. Ab dem ausgehenden 19. Jahrhundert wird diese Form von einer Poetik des Rätsels abgelöst. Das Rätsel setzt wie der Kasus beim konkreten Fall an, verweilt aber gewissermaßen beim Singulären, beim bloßen Problem der Detektion des Einzelfalls. Ich werde mit Rückgriff auf André Jolles' Typologie der Einfachen Formen ${ }^{11}$ zu zeigen versuchen, dass im Übergang vom Kasus zum Rätsel die Möglichkeit einer Kritik am Normsystem selbst problematisiert wird, dass also der Wandel in der Poetik der Kriminalliteratur auf eine historische Krise der Normkritik zurückgeführt werden kann.

Diese These soll im Folgenden an zwei exemplarischen Texten der Zeit belegt werden. Jodokus D.H. Temmes Kriminalgeschichte Wer war der Mörder? (1873) ist ein Text, der den Umbruch der Gattungspoetik in sich selbst sichtbar macht. So folgt er in der Darstellung eines ersten Mordfalls mit normkritischer Absicht noch ganz der Poetik des Kasus, um anhand eines zweiten Mordes die Probleme zu diskutieren, die sich aus einer kasuistischen Normkritik ergeben. Vor allem die Unzulänglichkeit einer Ermittlungspraxis, die nur noch das gepflegte Gespräch mit den Verdächtigen kennt, zeigt die Grenzen der Fallgeschichten auf, in denen sich der Kriminalfall wie von selbst auflösen muss. Auf dieses Problem reagiert Auguste Groner in der Detektiverzählung Der seltsame Schatten (1891), die wie die Kriminalgeschichten ihres ungleich bekannteren Zeitgenossens Arthur Conan Doyle der Poetik des Rätsels folgt, das von einem genialen Detektiv gelöst werden muss. Das Rätsel zeigt zwar einen Ausweg aus den Unzulänglichkeiten der tradi-

10 Als historischen »Kulminationspunkt« hat u.a. Joachim Linder das Jahr 1888 ausgemacht, das Jahr der Morde von Jack the Ripper und das Erscheinungsjahr von Arthur Conan Doyles A Study in Scarlet (Joachim Linder: Verbrechen erzählen. Zur literarischen Repräsentation von Kriminalität und Strafverfolgung vom 18. bis zum 21. Jahrhundert, in: ders.: Wissen über Kriminalität. Zur Medien- und Diskursgeschichte von Verbrechen und Strafjustiz vom 18. bis zum 21. Jahrhundert, hg. von Claus-Michael Ort, Würzburg 2013, S. 13-187, hier S. 21).

11 André Jolles: Einfache Formen. Legende/Sage/Mythe/Rätsel/Spruch/Kasus/Memorabile/ Märchen/Witz [1930], 2. Aufl., Tübingen 1958. 
tionellen Ermittlungspraxis, verliert dabei aber das Verhältnis von Legalität und Legitimität aus dem Blick und gibt so die Möglichkeit einer Kritik am Normsystem auf.

\section{Kasuistische Normkritik}

Jodokus Donatus Hubertus Temme (1798-1881) ist in der Kriminalliteraturforschung kein Unbekannter mehr. ${ }^{12}$ Für das 19. Jahrhundert nimmt er gleich in mehreren Diskursfeldern eine herausragende Stellung ein, war er doch zugleich Kriminalschriftsteller, Jurist und Politiker. Er macht in Preußen früh Karriere als Richter, Staatsanwalt und Strafverfahrensrechtler und wird während der 1848er Revolution Abgeordneter des preußischen Märzparlaments sowie der Frankfurter Paulskirchenversammlung. Sein Engagement für eine konstitutionelle Monarchie bringt ihm nach dem Scheitern der Märzrevolution einen Prozess wegen Hochverrats ein, den er zwar gewinnt, aber in der Folge durch den Einfluss der restaurativen Kräfte dennoch sein Amt verliert. Er emigriert nach Zürich, wo er sich als freier (Kriminal)Schriftsteller und zunächst unbesoldeter Rechtsprofessor durchschlägt und schließlich 1881 stirbt.

Temmes Tätigkeit als liberaler Politiker ist mit seiner rechtstheoretischen Haltung eng verbunden. Schon im Studium wurde er von der damals noch sehr jungen historischen Rechtsschule beeinflusst. ${ }^{13}$ Deren Hauptvertreter

12 Die erste positive Würdigung von Temme als belletristischen Schriftsteller stammt bereits aus dem Jahr 1914 (Max Gust: J.D.H. Temme. Ein münsterländischer Schriftsteller und Politiker des 19. Jahrhunderts, Diss., Münster 1914). Temme hat mehrere autobiographische Texte verfasst, die im Jahr 1883, kurz nach seinem Tod, unter dem Titel Erinnerungen herausgegeben wurden und inzwischen in einer Neuausgabe vorliegen Jodokus D.H. Temme: Augenzeugenberichte der deutschen Revolution 1848/49. Ein preußischer Richter als Vorkämpfer der Demokratie, neu hg. und mit einem Anhang versehen von Michael Hettinger, Darmstadt 1996). Seine Arbeit als Schriftsteller kommt hier allerdings nur am Rande zur Sprache, wohl auch, weil Temme seine kriminalliterarischen Texte selbst als Brotschriftstellerei abtut (vgl. ebd., S. 301-314). Zur Biographie Temmes vgl. Michael Hettinger: J.D.H. Temme (1798-1881) - nicht nur ein Juristenleben, in: Vom mittelalterlichen Recht zur neuzeitlichen Rechtswissenschaft. Bedingungen, Wege und Probleme der europäischen Rechtsgeschichte, hg. von Norbert Brieskorn u.a., Paderborn u.a. 1994, S. 335-364, sowie Karoline Peters: J.D.H. Temme und das preußische Strafverfahren in der Mitte des 19. Jahrhunderts, Berlin/New York 2010, S. 7-48.

13 Zum Verhältnis Temmes zur historischen Rechtsschule vgl. Karoline Peters: J.D.H. Temme, S. 272-285. 
Friedrich Carl von Savigny lehnt die aufklärerische Vorstellung ab, wonach sich Gesetze durch vernunftmäßige Rekonstruktion aus dem Naturrecht ableiten lassen. Er fordert, dass sich das geltende Recht nach dem >Volksgeist zu richten habe. Recht ist für die Vertreter der historischen Schule in erster Linie Gewohnheitsrecht und verlangt daher nach einer rechtshistorischen Betrachtung. Temme kritisiert an dieser Schule, dass sie zugunsten der historischen Fokussierung den gegenwärtigen Volksgeist vernachlässige. Er plädiert dafür, das gegenwärtige Rechtsbewusstsein zur Grundlage der Gesetzgebung zu erheben. Dann aber ergibt sich für ihn die politische Konsequenz, dass das Volk der Souverän in Fragen der Gesetzgebung sein muss und nicht etwa ein Monarch. Im Begriff der Volkssouveränität verbinden sich so seine rechtstheoretischen Überlegungen mit seiner staatsliberalen Haltung.

Die Auffassungen der historischen Rechtsschule haben direkte Konsequenzen für die Funktion der Falldarstellungen in der Kriminalliteratur. Solange die Autoren im Geiste der Aufklärung von einer transhistorischen und transkulturellen Geltung des Naturrechts ausgehen konnten, konnte auch der einzelne Fall als lehrhaftes Exempel dargestellt werden. Wenn das Recht jedoch nicht mehr in einer metaphysischen Instanz, in Gott, der Natur oder der Vernunft, verankert ist, dann gibt es kein allgemeingültiges System, aus dem sich ein singuläres Exempel deduzieren ließe, dann kann man aus Rechtsfällen nicht exemplarisch lernen. Dafür erhält jedoch der einzelne Fall systematisches Gewicht: Er wird zum Prüfstein für das Rechtsbewusstsein und dadurch überall dort zum notwendigen Medium einer Kritik am Gesetz, wo Legalität und Legitimität auseinandertreten.

Temmes besonderes Interesse als Rechtstheoretiker gilt der konkreten Entwicklung des Strafverfahrensrechts. ${ }^{14}$ In diesem Bereich haben sich zwischen der preußischen Kriminalordnung von 1805 und der Reichsstrafprozessordnung von 1877 große Veränderungen vollzogen, ${ }^{15}$ die tiefe Spuren in der literarischen Darstellung von Ermittlungspraxen hinterlassen haben. An die Stelle des Untersuchungsrichters, der Ankläger und Richter in einer Person ist, tritt die Staatsanwaltschaft als eigene Ermittlungsbehörde. Allmählich differenziert sich so die Ermittlung als eigenständiger Bereich des Strafverfahrensrechts mit eigenen Zuständigkeiten und eigenen Ermitt-

14 Im Detail informiert darüber Karoline Peters: J.D.H. Temme.

15 Einen Überblick über die Entwicklung des deutschen Strafprozessrechts im 19. Jahrhundert liefert Thomas Vormbaum: Einführung in die moderne Strafrechtsgeschichte, 2. Aufl. Berlin/Heidelberg 2011, S. 88-107. Vgl. Eberhard Schmidt: Einführung in die Geschichte der deutschen Strafrechtspllege, Göttingen 1947, §§ 254, 259f. u. 289-304. 
lungstechniken aus. Temmes Forderungen reichen dabei im Übrigen sogar weiter, als sie bis heute realisiert wurden. So forderte er, dass bereits die Voruntersuchung öffentlich sein und dass zwischen Staatsanwaltschaft und Beschuldigtem >Waffengleichheit gelten müsse. In Fragen der Ermittlungspraxis ging es ihm also vor allem darum, das Machtgefälle zwischen der staatlichen Ermittlungsbehörde und dem Individuum zu nivellieren.

Auch wenn Temme diese historischen Entwicklungen in seinen kriminalliterarischen Texten nicht explizit zur Sprache bringt, werden seine Erfahrungen als Politiker, Richter und Strafrechtstheoretiker doch in den Ermittlungen seiner Protagonisten ständig implizit verhandelt; und Temme war ein Kriminalautor von beeindruckender Produktivität. ${ }^{16}$ Alleine in der Gartenlaube ist er mit 34 Titeln vertreten. Sein CEuvre zählt insgesamt weit über 100 Texte, von denen hier nur ein exemplarischer Text herausgegriffen werden soll, der für die Poetik der Kriminalliteratur und für das Verhältnis von Tötungsarten und Ermittlungspraktik besonders aufschlussreich ist. Es handelt sich dabei um die Kriminalnovelle Wer war der Mörder? aus dem Jahr 1873.

Der Ich-Erzähler dieser Novelle ist Kriminaldirektor und damit oberster Untersuchungsrichter einer ungenannten Provinzstadt. Die Handlungszeit muss also vor der Einführung einer Staatsanwaltschaft liegen (in Berlin wurde die Staatsanwaltschaft erstmals 1846 eingesetzt, 1849 wurde die neue Regelung auf ganz Preußen ausgedehnt). Temme war selbst ab 1836 für zwei Jahre Kriminaldirektor in Stendal, wo er an die preußische Kriminalordnung von 1805 gebunden war. ${ }^{17} \mathrm{Zu}$ dieser Zeit verfasste er auch einen kritischen Kommentar über diese Kriminalordnung, der 1838 erschien. ${ }^{18}$ Es lässt sich also vermuten, dass Temme seine Erfahrungen aus dieser Zeit in der Novelle verarbeitet.

In der Erzählung gibt es eigentlich zwei Mordfälle. Der erste wird auf gerade einmal zweieinhalb Seiten abgehandelt. Es beginnt damit, dass ein alter Bauer den Ich-Erzähler aufsucht und von ihm Gerechtigkeit für seinen Sohn einfordert, der von einem Forstbeamten erschossen wurde:

16 Vgl. dazu die umfassende Werkbibliographie in dem Art. Jodokus Donatus Hubertus Temmer, in: Westfälisches Autorenlexikon, hg. von Walter Gödden und Iris Nölle-Hornkamp, 4 Bde., Paderborn 1993-2002, Bd. 1, S. 305-323.

17 Die zentralen Paragraphen der preußischen Kriminalordnung sind abgedruckt in Wolfgang Sellert/Hinrich Rüping: Studien- und Quellenbuch zur Geschichte der deutschen Strafrechtspllege, Aalen 1989, Bd. 1, S. 501-508.

18 Jodocus D.H. Temme: Commentar über die wichtigeren Paragraphen der Preußischen Criminalordnung. Zunächst für preußische Inquirenten, Berlin 1838. Zur preußischen Kriminalordnung und Temmes Kritik vgl. Karoline Peters: J.D.H. Temme, S. 77-94. 
Es war ein sehr einfacher Hergang, den er mir mitteilte. Vorfälle solcher Art kamen damals leider des Öfteren vor; nicht bloß in jenen Revieren, nicht bloß in jener Provinz. Ein trauriges Gesetz hatte sie im ganzen Lande hervorgerufen. Der alte Bauer hatte geschlagenes Holz in dem königlichen Forst gekauft. Sein Sohn war mit einem Knecht hingefahren, um es aufzuladen und abzuholen. Während sie beim Aufladen waren, war der Revierförster hinzugekommen. Es war Holz an mehrere Personen verkauft worden. Die verschiedenen verkauften Haufen lagen in Reihen beisammen. Der Förster behauptete, der Sohn des Bauern lade einen fremden größeren Haufen auf, und verlangte, wieder abzuladen. Der junge Bauer behauptete in seinem Recht zu sein. Der Förster bestand auf seinem Befehl und erklärte, ihn mit Gewalt durchsetzen zu müssen und zu wollen. Der junge Bauer entgegnete, Gewalt mit Gewalt zurückweisen zu wollen. Der Förster kam näher. Der Bauer streckte ihm drohend seine Axt entgegen. Der Förster legte sein Gewehr auf ihn an und forderte ihn auf, die Axt aus der Hand zu legen. "Nimmer! « rief der Bauer. Der Förster wiederholte die Aufforderung; er werde sonst schießen. Das möge er einmal wagen, ruft der Bauer, seine Axt schwingend. Der Förster schießt den jungen Bauern nieder. ${ }^{19}$

Schon der juristische Stil des Berichts lässt erahnen, dass hier ein Rechtsproblem thematisiert werden soll: Anlass des tödlichen Streits ist der Verdacht des Försters, dass der Bauernsohn Holz stehlen will. Allerdings schwächt der Text diesen Verdacht in größtem Maße ab, weil der vermeintliche Diebstahl lediglich darin besteht, dass der Bauer mehr Holz mitnehmen will als er bezahlt hat und im Übrigen wohlhabend genug wäre, um mehr zu kaufen. Dass es aber überhaupt um einen Holzdiebstahl geht, ist signifikant. Denn dabei handelt es sich um ein Vergehen, das seit der Frühen Neuzeit als Paradebeispiel eines ssocial crime (Eric Hobsbawm) fungiert. Als solches verbirgt sich hinter der Tat der soziale Konflikt zwischen Untertanen und Feudalherrschern, die ihr landesherrliches Forstrecht ausüben. ${ }^{20}$

In Temmes Novelle muss der Ich-Erzähler den Vater des jungen Bauern darüber aufklären, dass der Förster tatsächlich in Einklang mit dem Gesetz gehandelt hat: Die restaurative Gesetzgebung des 19. Jahrhunderts ermöglicht nicht nur das harte Durchgreifen der Forstbeamten, es verpflichtet sie geradezu dazu. Der Vater reagiert entsprechend entsetzt: "In den alten Augen gesellten sich zu den Tränen des Schmerzes Tränen des Zornes. >Welche Gesetze! Welches Recht! , rief er. ${ }^{21}$ Der Text weist hier schon durch

19 Jodokus D.H. Temme: Wer war der Mörder?, in: ders.: Wer war der Mörder? Kriminalgeschichten, hg. von Günther Butkus, Bielefeld 2010, S. 143-180, hier S. 143f.

20 Vgl. Heinrich Rubner/Friederike von Gadow: 'Forst, Forstrecht, in: Handwörterbuch zur deutschen Rechtsgeschichte (HRGdigital), hg. von Albrecht Cordes, Heiner Lück und Dieter Werkmüller, http://www.hrgdigital.de/HRG.forst_forstrecht (Stand: 27.9.2014).

21 Jodokus D.H. Temme: Wer war der Mörder?, S. 144. 
seine Affektpoetik - der Zorn über unrechtes Recht - auf den Widerspruch zwischen Legalität und Legitimität, zwischen Gesetz und Rechtsbewusstsein des Bauern hin. Dem schließt sich der Ich-Erzähler mit explizitem Verweis auf das Rechtsbewusstsein an. Könnte sich nämlich, so der Ich-Erzähler, »doch noch ein Umstand ermitteln lassen, der die offenbare Roheit, die jedenfalls vorlag, auch als eine ungesetzliche, strafbare Handlung darstellte! Dem verletzten allgemeinen Rechtsbewusstsein gebührte dann die strengste rechtliche Genugtuung. ${ }^{22}$ Wie häufig in seinen Kriminalerzählungen verfolgt der Rechtstheoretiker Temme im Sinne seiner Interpretation der historischen Rechtsschule das didaktische Ziel, das Rechtsbewusstsein seiner Leser zu stärken ${ }^{23}$ und dadurch die Rechtslage zu kritisieren.

Die poetische Form der Fallgeschichte, die Temme verwendet, ist seit dem ausgehenden 18. Jahrhundert, seit den Kriminalerzählungen Meißners und Schillers bekannt. Die Erzählung eines singulären Kasus erfüllt seither die soziale Funktion, Differenzen zwischen Legalität und Legitimität aufzuzeigen - im vorliegenden Beispiel also das Problem, dass die summa iniuria, die "offenbare Roheit« der Tat, das summum ius ist, also gerade keine "ungesetzliche, strafbare Handlung « darstellt. Der Kasus zielt dabei, wie André Jolles in den Einfachen Formen herausgearbeitet hat, auf »etwas unbedingt Strafbares, was von Paragraphen unabhängig, durch sie nicht zu fassen ist«, mithin auf »jenes Unrecht, das nicht von einer Norm bedingt ist, das absolute Unrecht. ${ }^{24}$ Und diese Zielvorgabe einer Kritik der Rechtsstruktur durch das absolute Unrecht ist es auch, die die Ästhetik des Schreckens der kriminalliterarischen Fallgeschichten legitimiert, weil sie umso besser funktionieren, je abschreckender sie das Verbrechen schildern. ${ }^{25}$

22 Ebd.

23 Vgl. dazu Winfried Freund: Demokrat, Richter, Kriminalautor. Eine Wiederbegegnung mit Jodokus Donatus Hubertus Temme, in: Autoren damals und heute. Literaturgeschichtliche Beispiele veränderter Wirkungshorizonte, hg. von Gerhard P. Knapp, Amsterdam/Atlanta, GA 1991, S. 257-271, hier S. 267.

24 André Jolles: Einfache Formen, S. 174.

25 Mit der These dieser sozialen Funktion soll nicht bestritten werden, dass die Fallgeschichte der Spätaufklärung zusätzlich eine anthropologische Funktion erfüllt, die für die Autoren der Zeit um 1800 vielleicht sogar wichtiger ist. Doch bereits sie thematisieren die politische Funktion ihrer Kriminalerzählungen, etwa wenn Meißner auf die Unterscheidung von "gesezlicher und moralischer Zurechnung" abzielt (August Gottlieb Meißner: Vorrede [zur 13. und 14. Sammlung der "Skizzen«, 1796], in: ders.: Ausgewählte Kriminalgeschichten. Mit einem Nachwort hg. von Alexander Košenina, St. Ingbert 2003, S. 7-11, hier S. 10) und wenn Schiller »die republikanische Freiheit des lesenden Publikums, dem es zukömmt, selbst zu Gericht zu sitzen« befördern will (Friedrich Schiller: Der Verbrecher aus verlorener Ehre. Eine wahre Geschichte, in: Schillers Werke. Nationalausgabe, begründet von Julius Petersen. Weimar 1943ff., Bd. 16, S. 7-29, hier S. 8). 
Im Übergang von den frühmodernen didaktischen Kriminalberichten zum Kasus kehrt sich die moralische Bewertung um. Während im 17. und frühen 18. Jahrhundert zumeist die Rechtmäßigkeit des Normsystems und die Unrechtmäßigkeit des Normbruchs ausgestellt werden, mithin Legalität und Legitimität eine kompakte Einheit bilden, sympathisieren die Autoren der spätaufklärerischen Fallgeschichten mit dem Normbruch, um die Unrechtmäßigkeit der Gesetze aufzuzeigen. Es geht, um noch einmal Jolles zu zitieren, um ein »Messen von Maßstab an Maßstab « ${ }^{26}$ Und dieser zweite Maßstab, an dem sich der erste Maßstab, also das positive Recht, messen lassen muss, ist für Temme ebenjenes Rechtsbewusstsein, das sich in der moralischen Entrüstung eines alten Bauern zeigt.

\section{Ermittlungsprobleme}

Nun schreibt Temme allerdings ein Jahrhundert nach Schiller; der Kasus in seiner idealtypischen Form ist bei ihm nur im ersten Mord sichtbar. ${ }^{27}$ Bei dem zweiten Mordfall aber, der im Mittelpunkt der Erzählung steht, kommt die Ermittlungspraxis ins Spiel. Als der Erzähler den Förster befragt - er heißt übrigens 'Wolf^, wie der Protagonist aus Schillers Verbrecher aus verlorener Ehre -, hinterlässt dieser nur den Eindruck »des furchtbarsten Fanatismus ${ }^{28}{ }^{8}$ Der Leser kann hier bereits ergänzen, dass nur durch einen solchen Gesetzesfanatismus das allgemeine Rechtsbewusstsein so unterdrückt werden kann, dass der Förster alleine nach "amtliche[r] Autorität« und "Pflicht« handelt. ${ }^{29}$ Die Verbindung von kaltblütiger Pflichterfüllung und glühendem Fanatismus lässt Wolf wie ein Exempel für die Dialektik der Aufklärung erscheinen.

$\mathrm{Zu}$ denken gibt dem Ermittler aber nicht nur die verdächtige Person Wolfs, sondern auch eine Reihe von Rätseln und Geheimnissen: Da ist die schöne und junge Frau des abstoßenden Försters, Marianne, die einen rätselhaft

26 André Jolles: Einfache Formen, S. 179.

27 Die Distanzierung von der Fallgeschichte über die Problematisierung der Ermittlungsarbeit hat Joachim Linder: Verbrechen erzählen, S. 135-141, an einer anderen Erzählung Temmes mit dem Titel Rosa Heisterberg nachgewiesen. Hans-Otto Hügel sieht bei Temme bereits die Frage nach (oder im Sinne dieses Beitrags formuliert: das Rätsel von) dem Täter als neue Erzählform auftauchen (Hans-Otto Hügel: Untersuchungsrichter, Diebsfänger, Detektive, S. 149-161 u. 206).

28 Jodokus D.H. Temme: Wer war der Mörder?, S. 149.

29 Ebd. 
kränklich-traurigen Eindruck hinterlässt. Da ist der väterliche, aber wortkarge Knecht Mariannes, der ihr verschworene Blicke zugeworfen hat. Und da ist das hölzerne Kreuz am Wegrand, das die Stelle markiert, an der vor sieben Jahren der zweite Mord geschehen war. Opfer war Alfred Felsener, der als Forsteleve im Forsthaus gewohnt hatte. Allem Anschein nach hat es sich um eine für die Fallgeschichten der Spätaufklärung typische Tötungsart gehandelt: Der Forsteleve soll einen Wilddieb überrascht haben und von diesem erschossen worden sein.

Wenige Tage nach dem Vorfall bekommt der Erzähler Besuch vom Gefängnisgeistlichen, der ihm berichtet, dass ein alter Sträfling im Sterben liegt. Bei diesem handelt es sich just um die Person, die für den Mord an dem Forsteleven Felsener sieben Jahre zuvor verurteilt wurde, die aber seitdem und noch auf dem Totenbett die Tat bestreitet. Also rollt der Erzähler den alten Fall noch einmal auf. Ausführlich schildert der Text seine Ermittlungen: Aus den Akten erfährt er, dass der Täter aufgrund eines Indizienprozesses überführt wurde. Er selbst hatte angegeben, dass er kurz vor der Tatzeit nicht etwa vom Forsteleven Felsener, sondern vom ehemaligen Förster, dem Vater Mariannes, bei einem Wilddiebstahl überrascht worden sei. Dieser habe sein Gewehr entwendet und den Eleven erschossen. Bei den Untersuchungen war man zwar diesem Verdacht nachgegangen, es stellte sich aber heraus, dass noch eine Stunde vor der Tat niemand das Forsthaus verlassen hatte, man für den Weg zwischen Forsthaus und Tatort aber eineinhalb Stunden benötigt; und so hielt man die Anschuldigungen des Wilddiebes für widerlegt.

Die neuen Ermittlungen des Erzählers beschränken sich fast ausschließlich auf das Verhör; und die Vernehmung des alten Knechts bringt ihm eine entscheidende Information, die plötzlich die Version des Wilddiebs plausibel macht: Es gibt nämlich einen geheimen zweiten Weg vom Forsthaus zum Tatort, der deutlich kürzer ist. Dadurch wird zwar nicht der damals bereits gichtkranke Förster, wohl aber dessen Tochter Marianne und der Jäger Wolf verdächtig. Tatsächlich stellt sich heraus, dass der vermeintliche Täter den Jäger Wolf mit Mariannes Vater in der Dunkelheit verwechselt hat. Gerade als der Erzähler die Frau verhört und kurz vor einem Geständnis steht, löst sich der Fall von selbst. Als der Jäger Wolf von den Verhören erfährt, tötet er sich selbst, und Marianne gesteht, dass ihr Mann es war, der vor sieben Jahren den Forsteleven aus Eifersucht erschossen hat.

Die poetische Form hat sich hier deutlich gewandelt. Es geht nicht mehr um die Kritik an einem ungerechten Feudalrecht, sondern um das Problem der richtigen Detektion. Der Kasus konnte die Frage der erfolgreichen Ermitt- 
lung ausklammern, weil der Hauptverdächtige sich für gewöhnlich als Täter entlarvt. Im ersten Mordfall musste Temmes Erzähler noch nicht einmal ermitteln; Täter und Tathergang standen von Beginn an fest. Bei dem zweiten Mord ist der Protagonist hingegen als Kriminaldirektor gefordert. Das strukturelle Problem seiner Ermittlung besteht darin, dass er keine peinliche Befragung durchführen kann, im Gegenteil: In seinen Verhören vermeidet er jedes Moment staatlicher Macht. Als der alte Knecht in der Vernehmung den Entschluss fasst, den Namen des Täters für sich zu behalten, kann der Erzähler nur anerkennend zugestehen: "[E]s war etwas Großes in diesem Entschluß des alten, an seinen Gott, an ein ewiges Leben, an Belohnung und an Strafe in jenem Leben glaubenden Mannes[.] Er wollte die Strafe auf sich nehmen, aber nicht zum Verräter werden. $\ll^{30}$ Dem Erzähler bleibt nur der Abbruch des Verhörs.

Noch hilfloser wirkt er bei der Vernehmung Mariannes. Es ist ihm ein "saurer Weg « ${ }^{31}$ das »Herz" will ihm »brechen" und sein "Amt « erscheint ihm »in diesem Augenblick so schwer ${ }^{32}$ Er empfindet »[e]ine leise Freude, ein[en] klein[en] Triumph", als Marianne die Fassung behält und seine Fragen nicht beantwortet, und er ist sich der Paradoxie in dieser Situation bewusst, wenn er resümiert: "Das Herz des Menschen muß manchmal den Beruf verleugnen. ${ }^{33}$ In diesem Widerspruch zwischen Herz und Pflicht des Ermittlers, der ihn von der kaltblütigen Pflichterfüllung des Försters abhebt, manifestiert sich das ganze Problem seiner Strafverfolgung. Und so bricht er auch diese Vernehmung ab:

"Madame«, sagte ich, »ich verzichte auf fernere Fragen an Sie. Ich bedaure nur, daß ich Ihnen Ihr Leiden nicht abnehmen kann. Doch eine Bitte habe ich noch. Sollte einmal dieser Druck, der auf Ihnen lastet, zu schwer für Sie werden - die Stunde kann kommen mit der ganzen zwingenden Gewalt der Wahrheit und des Rechts, dann werden Sie in mir zwar den Richter, aber auch den Freund finden, der soviel es in seinen Kräften steht, sie trösten und aufrichten wird.« ${ }^{34}$

Man sieht: Dieser Inquirent ist durch die Schule der Empfindsamkeit gegangen. Temmes Protagonist erfüllt alle Forderungen, die der Autor in seinen juristischen Schriften zur staatlichen Ermittlung verlangt: Die >Waffengleichheit` zwischen Beschuldigtem und Untersuchungsrichter ist hergestellt. Au-

${ }^{30}$ Ebd., S. 173.

31 Ebd.

32 Ebd., S. 175.

33 Ebd., S. 177.

34 Ebd., S. 178. 
ßerdem verzichtet der Ich-Erzähler auf das (für Temme moralisch untragbare) Vorgehen, das Vertrauen des Verdächtigen zu erschleichen, um an kompromittierende Informationen zu gelangen. ${ }^{35}$ Nur wie soll der Ermittler dann einen Täter überführen, der ihm nicht freiwillig gesteht? Bei Schiller hat sich dieses Problem noch von selbst gelöst. Sein Protagonist im Verbrecher aus verlorener Ehre gesteht, sobald er an einen Oberamtmann gerät, der ihm mit "Anstand und Mäßigung « begegnet. ${ }^{36}$ Weil Temmes Verbrecher sich aber weigert, seine Humanität durch das Geständnis des Rechtsbruchs triumphieren zu lassen, erweist sich die empfindsame Verhörtechnik als unzureichend. Sie endet, um einen Begriff Michael Niehaus' zu verwenden, im »Leerlauf der Literaturinquisition«. ${ }^{37}$ Und sie zeigt das Problem einer Ermittlungspraxis, die sich ganz auf die Verhörpsychologie verlassen muss, weil sie nicht mehr auf die Folter (die in Preußen ja bereits 1740 abgeschafft worden war) und noch nicht auf die Methoden der modernen Kriminalistik zurückgreifen kann.

Temme bemüht sich, diesen Bruch zu kaschieren. Die letzten Worte des Ich-Erzählers haben einen so großen Effekt, dass Marianne schließlich doch gestehen will. Genau in diesem Moment nimmt ihr der Text das Geständnis ab: Die Nachricht vom Selbstmord ihres Mannes trifft ein. Die Lösung kommt - wie oft in Temmes Geschichten - von außen; sie gibt sich damit als prekär zu durchschauen, weil sie nur noch durch den literarischen Kunstgriff des sdeus ex machinar gelingen will. Doch selbst wenn an dieser Stelle die Ermittlungstechnik des Erzählers erfolgreich gewesen wäre, läuft der Text doch auf einen Widerspruch hinaus: Der Erzähler will, wie er Marianne gegenüber sagt, Richter und Freund zugleich sein, aber diese beiden Rollen sind eben nicht miteinander vereinbar, weil der Richter auf Seiten des allgemeinen Gesetzes stehen muss, der Freund aber auf Seiten des delinquenten Individuums. Im Kasus musste der konkrete Fall gelöst sein, weil sich aus ihm nur so die Kritik am abstrakten Recht ableiten ließ. Das Problem der erfolglosen Ermittlungspraxis zerschneidet das Band zwischen konkretem Fall und abstraktem Recht. Im Moment, in dem der Weg vom Einzelfall zur Rechtsnorm verstellt ist, stürzt die Form des Kasus als Medium der Rechtskritik in sich zusammen.

35 Dieses Vorgehen, auf das nach Abschaffung der Folter viele Untersuchungsrichter notgedrungen ausweichen mussten, moniert Temme explizit in seiner Kritik an der preußischen Kriminalordnung (Jodocus D.H. Temme: Commentar, S. 95, vgl. Karoline Peters: J.D.H. Temme, S. 87f.).

36 Friedrich Schiller: Der Verbrecher aus verlorener Ehre, S. 28.

37 Michael Niehaus: Das Verhör. Geschichte - Theorie - Fiktion, München 2003, S. 401. 
Temmes Kriminalerzählungen sind in der hier vorgestellten historischtypologischen Perspektive Übergangstexte. Sie enthalten noch Momente der juristischen Fallgeschichte, aber sie haben nicht mehr den Bezug zu einem rational begründeten Naturrecht, sie thematisieren bereits das Problem der Detektion, aber verfügen noch nicht über erfolgreiche Ermittlungspraktiken. Dieser letzte, detektivische Typus lässt sich in seiner ausgeprägten Form in der deutschsprachigen Literatur erst in den Jahren nach Temmes Tod beobachten. Exemplarisch dafür ist das Werk der österreichischen Autorin Auguste Groner, die zu Lebzeiten womöglich noch bekannter war als Temme, aber schon biographisch ein Gegenmodell zu diesem darstellt. ${ }^{38}$ Die Wienerin Groner, die von 1850 bis 1929 gelebt hat, war Volksschullehrerin und macht parallel eine beeindruckende Karriere als Schriftstellerin. Sie schreibt mehrere hundert Erzählungen und Romane, ist eine vielgelesene Volks- und Jugendautorin und wird vor allem für ihre Kriminalgeschichten bewundert. ${ }^{39}$ Ihre amerikanische Übersetzerin Grace Isabel Colbron zählt sie 1909 zu den weltweit bedeutendsten Autoren von Detektivgeschichten. ${ }^{40}$ Ihren festen Platz in den Literaturgeschichten hat sie vor allem, weil sie im Jahr 1889 mit der Figur des Joseph Müller den ersten Seriendetektiv in deutscher Sprache erfindet, der typologisch in großer Nähe zu Sherlock Holmes steht. Das ist auch deswegen bemerkenswert, weil Arthur Conan Doyles $A$ Study in Scarlet zwar kurz zuvor erschien, die erste deutsche Übersetzung allerdings erst seit 1894 auf dem Markt war. Man kann also mit vollem Recht von einer Parallelerfindung sprechen. Als Auguste Groner 1929 stirbt, hat sie an die 20 Erzählungen und Romane um ihren Detektiv geschrieben.

Wo bei Temme seine Arbeit als Jurist einen Deutungskontext für sein kriminalliterarisches Werk vorgibt, ist es bei Groner ihre Tätigkeit als Verfasserin von Zeitungstexten. Für die Zeitung ihres Mannes hat sie mehrfach Rätselgedichte geschrieben, auf die ein Geldpreis ausgeschrieben war. Ein

38 Zur Biographie Groners mit Blick auf die häufig unterschätzte Bedeutung von Autorinnen für die Geschichte der Detektivliteratur vgl. Evelyne Polt-Heinzl: Die Frau mit den vielen Krimis. Auguste Groner (1850-1929), in: dies.: Zeitlos. Neun Porträts. Von der ersten Krimiautorin Österreichs bis zur ersten Satirikerin Deutschlands, Wien 2005, S. 31-51.

$39 \mathrm{Zu}$ Groner liegt eine im Privatdruck erschienene Bibliographie vor: Gerhard Lindenstruth: Auguste Groner (1850-1929). Eine illustrierte Bibliographie, Gießen 1992.

40 Vgl. Grace Isabel Colbron: The Detective Story in Germany and Scandinavia, in: The Bookman 30/4 (1909), S. 407-412. Außerdem wird Groner für ihr literarisches Schaffen ausgezeichnet, bei der Weltausstellung in Chicago 1893 sowie bei der Kaiser-JubiläumsAusstellung in Wien 1898. 
kleines Beispiel davon findet sich in der Ausgabe vom 30. April 1896 in Das interessante Blatt, dessen Chefredakteur Groners Mann war. Der Verfasser des Rätsels zeichnet mit dem Namen "Renorga«, ein Palindrom, hinter dem sich der Name 'A. Groner verbirgt. Schon das Pseudonym der Autorin hat also eine Rätselform. In dem Rätselgedicht ist ein Wort mit zwei Silben gesucht:

Es kommt heran, es geht vorüber

Und ist doch allzeit überall.

Es ist, will sich's zum Übel wenden,

Verbunden stets mit einem Fall.

Es sendet, wie es große Herren

$\mathrm{Zu}$ thun belieben, Boten aus,

Dann bleibt, wenn es nur immer möglich,

Es nicht zu treffen, man zuhaus.

Viel Heere steh'n in seinem Solde,

Gar dräuend und gar ungestüm,

Sie führen mit ein unsichtbares,

Ein feuerspeiend Ungethüm.

Dies hat viel Übel schon verschuldet,

Verwandelt Leben oft in Tod

Und bringt, wohin sich's auch mag wenden,

Viel Angst hin, Schrecken, Gram und Noth.

Auch eisige Geschosse führt es

Mit sich, vernichtend manches Gut

Und sendet über blüh'ndes Leben,

Todbringend seine wilde Flut.

Ist wie ein Weib, das voller Launen,

Bald lacht und scherzt, bald zankt und weint,

Wie eine Mutter, die jetzt tröstet,

Jetzt mit dem bösen Kinde greint.

Ist wie ein Mann, der vielbeschäftigt,

Gereizt oft ist und voll Verdruss,

Der aber dennoch meist genau weiß

Das, was er soll und was er muss.

Und nur Gesetzen, unumstößlich,

Gehorchet es zu jeder Stund'

Und thut, wie alles Große, Mächt'ge,

Aus unsres Schöpfers Urkraft kund. ${ }^{41}$

41 Renorga [i.e. Auguste Groner]: Der Hundert-Guldenpreis des "Interessanten Blattes", in: Das interessante Blatt, 15. Jg., Nr. 18 (30. April 1896), S. 11. 
Wer auf das Lösungswort >Wetter gekommen und eine Postkarte an den Verlag geschickt hat, konnte sich als einer von 2.500 richtigen Einsendern eine Chance auf den Hauptgewinn von 100 Gulden ausrechnen.

Wie lässt sich nun dieses lyrische Preisrätsel als Interpretament für Groners kriminalliterarische Texte verwenden? Die Grundidee der folgenden Deutung besteht darin, dass das Rätsel genau diejenige poetische Form ist, die Groner auch ihren Kriminalnovellen zugrunde legt. Jolles zählt das Rätsel wie den Kasus zu den reinfachen Formen und argumentiert, dass es sich von seiner Frage-Antwort-Struktur her erklären lässt. Für ihn ist das Rätsel ein paradoxes Gebilde, weil es eine Frage stellt, die Antwort auf diese Frage aber in sich selbst enthält. ${ }^{42}$ Der Leser muss zwar die Indizien noch in einen Zusammenhang bringen, er muss beispielsweise wissen, dass es sich bei den »eisige[n] Geschosse[n]《 um Hagel handelt und dass schlechtes Wetter ein Tiefdruckgebiet voraussetzt, aber alle nötigen Hinweise zur Auflösung sind im Text bereits vorhanden.

Genau diese Struktur liegt Groners »Criminalgeschichte« Der seltsame Schatten (1891) zugrunde, an der sich die Poetik des Rätsels exemplarisch beobachten lässt. ${ }^{43}$ Der Text beginnt folgendermaßen:

Das Wochenblatt der Kreisstadt O. brachte folgende Notiz:

»Rätselhafter Mord. Sonntag, am Morgen des 10. Oktober, also vorgestern, wurde der pensionierte Polizeikommissar, Herr Anton Werner, ermordet in seinem Bett aufgefunden. Ein sicher geführter Stoß mit irgendeinem dolchartigen Instrumente hat ihn getötet.

Bis zur Ausgabe dieser Nummer, also bis heute mittag, ist es noch nicht gelungen, auch nur die geringste Spur des Mörders aufzufinden; ja, es ist noch nicht einmal gelungen, zu entdecken, was den Mord veranlaßte. Ein Raub liegt nicht vor. Feinde besaß das hochachtbare, menschenfreundliche Opfer dieses Verbrechens nicht. Somit ist letzteres bis jetzt unerklärt. Hat vielleicht Irrsinn die Tat begangen?

Und noch ein Rätsel! Es konnte bis jetzt, trotz allen Scharfsinns unserer so überaus gut geschulten Sicherheitsorgane, nicht einmal festgestellt werden, welchen Weg der Mörder gekommen, welchen er gegangen ist. [...]«4

Das Rätsel ist vom ersten Moment an auf mehreren Ebenen präsent. Zwar hat bereits Temmes Erzähler von Rätseln und Geheimnissen gesprochen,

42 Vgl. André Jolles: Einfache Formen, S. 129.

43 Auguste Groner: Der seltsame Schatten, in: Prochaska's illustrirte Monatsbände 2/9 (1891), S. 63-130. Zitiert wird nach der Ausgabe Auguste Groner: Der seltsame Schatten, in: dies.: Der Brief aus dem Jenseits. Kriminalnovellen, 2. Aufl., Berlin 1986, S. 137198. Bei dieser Erzählung handelt es sich - trotz typologischer Nähe - um keinen ihrer Joseph-Müller-Krimis.

44 Auguste Groner: Der seltsame Schatten, S. 137. 
aber er meinte damit Verhaltensbeobachtungen, die er sich nicht erklären konnte. Bei Groner ist das Rätsel komplexer. Zunächst liegt es in Form einer (inzwischen klassisch gewordenen) locked room mistery vor. Wie der Leser im Verlauf der Erzählung genauer erfährt, war das Zimmer abgeschlossen, der Schlüssel steckte von der Innenseite und nach den Gewohnheiten des Opfers war das innere Fenster angelehnt, das äußere aber, in das ein kleines Windrad eingearbeitet war, fest verschlossen. Niemand im Haus hat einen Eindringling bemerkt. Zudem liegt das Rätsel als Frage nach dem Mörder, als Whodunnit? vor, denn es ist rätselhaft, wer überhaupt ein Motiv gehabt haben kann, das Opfer zu töten. So wird sogar ein Mord aus »Irrsinn« in Betracht gezogen, weil nur eine unzurechenbare Tat unerklärlich bleiben darf. Schließlich aber ist auch die Tötungsart selbst rätselhaft. Der tödliche Stoß mit »irgendeinem dolchartigen Instrument« steht in keinem Zusammenhang mit einem ssocial crimer wie Temmes Holz- oder Wilddiebstahl. Es zählt also nicht zu der Art von Verbrechen, deren Darstellung für die Normkritik im Kasus hochgradig funktional war. Tatort ist auch nicht der außersoziale Raum eines Waldes, sondern ein Privatraum innerhalb einer Stadt folglich gibt es eine extrem hohe Zahl von potentiellen Verdächtigen. Die Ermittlungspraxis kann sich schon deswegen nicht ausschließlich auf das Verhör beschränken.

Wenige Tage nach dem Mordanschlag taucht ein Geheimpolizist bei der Familie des Opfers auf, der so vorgeht, wie man es heute von einem Detektiv erwartet. So versucht er, den Schattenfall zu rekonstruieren, den die Haushälterin in der Tatnacht gesehen haben will, er untersucht das Fenster, überprüft die Länge der Leitern im Garten und entdeckt eine Drahtspule, die in einen Brief eingewickelt ist. Als genialer Detektiv findet er heraus, dass der Schatten von einer pendelnden Lampe neben dem Fenster gekommen sein muss und er folgert, dass der Dieb mithilfe beider Leitern durch das Fenster ein- und ausgestiegen ist. Die Drahtspule gibt ihm den Hinweis, dass der Täter mit einem stabilen Draht durch das Windrad gegriffen und so das Fenster entriegelt und zugesperrt hat. Schließlich bringt ihn der Brief auf die Spur eines Wiener Uhrmachers, bei dem er eine Uhr entdeckt, die sich bis zum Tod des Opfers in dessen Besitz befunden haben muss. Der Uhrmacher zeigt sich recht bald von der "fixe[n] Idee « ${ }^{45}$ besessen, ein Erfinder zu sein, und der Detektiv findet sogar heraus, dass er den Raubmord nur begangen hat, um den Mechanismus der Uhr des Opfers zu studieren. Damit ist also selbst der Wahnsinn in die Kausalerklärung eingebettet.

45 Ebd., S. 184. 
In Groners Detektivgeschichte ist die Tat das Rätsel, das vom Detektiv gelöst wird. Allerdings stellt sich in der Novelle heraus, dass der Detektiv auf eigene Faust ermittelt und für die Polizei eigentlich der Hauptverdächtige ist. Das Rätsel, das er löst, hat also genau genommen die poetische Form des Halsrätsels, bei dem das Leben des Ratenden auf dem Spiel steht und das seit dem Rätsel der Sphinx zu einer idealtypischen Rätselform geworden ist. Die Übertragung des Halsrätsels vom Mythos zur Kriminalliteratur bewirkt aber, dass dort nicht mehr die Frage nach der Norm, sondern nach der Ermittlung, und das heißt: nach der Lösungsstrategie des Rätsels gestellt wird. Die Verurteilung droht nicht als Strafe für eine gebrochene Norm, sondern für die gescheiterte Ermittlung.

Die Detektiverzählung mit der poetischen Form des Rätsels zu beschreiben, ist nicht neu. Schon Brecht hat den Kriminalroman in die Nähe des Kreuzworträtsels gestellit ${ }^{46}$ und Jolles meinte, dass sich »das Rätsel des Verbrechens [...] in der Neuzeit« zur "Detektiverzählung« entwickelt hat. ${ }^{47}$ Dieser Auffassung hat allerdings Richard Alewyn widersprochen. Er schreibt:

Gewiß, auch im Rätsel wird gefragt, aber niemals nach einem Individuum, sondern immer nur nach einer Gattung und nicht nach einer unbekannten, sondern nach einer allbekannten Gattung. Die Lösung des Rätsels der Sphynx lautet: der Mensch. [...] Die Frage des Detektivromans dagegen bezieht sich immer nur auf Individuelles $[\ldots] \ll{ }^{48}$

Dieser Einwand hat einiges für sich. Denn tatsächlich geht es in den Rätseln insofern um Gattungen, als das zu Erratende über eine Analyse seiner Bestandteile erschlossen werden muss. Doch das Individueller tritt im Detektivroman (wie im Rätselgedicht) ja immer als Bündel von Kennzeichnungen

46 Vgl. Bertolt Brecht: Über die Popularität des Kriminalromans [1938/1940], in: Der Kriminalroman, S. 33-37, hier S. 33.

47 André Jolles: Kleine Formen, S. 148.

48 Richard Alewyn: Anatomie des Detektivromans, S. 61. Als weitere, einflussreiche Kritik der Rätseltheorie ist Hans-Otto Hügels Standardwerk zur deutschen Detektiverzählung im 19. Jahrhundert zu nennen (Hans-Otto Hügel: Untersuchungsrichter, Diebsfänger, Detektive, S. 3-23). Allerdings weist Hügel genau betrachtet nur eine allzu enge Auslegung des Rätselbegriffs zurück, wie sie beispielsweise Brechts unglücklich gewählte Analogie des Kreuzworträtsels nahelegt, und kritisiert diejenigen Kulturtheorien, die die Rätselform nur als Teil einer Rationalisierungstheorie im Prozess der Modernsierung begreifen wollen. Der Sache nach ist er aber von einem poetologisch verstandenen Rätselbegriff nicht so weit entfernt, der sich zudem wohl besser zur Beschreibung eignet als der von ihm verwendete Begriff von der Detektion als 'Arbeit< (zur Kritik an der systematischen Überlastung des Arbeitsbegriffs vgl. Ulrich Schulz-Buschhaus: Hans-Otto Hügel: Untersuchungsrichter - Diebsfänger - Detektive [...] [Rezension], in: Arcadia 16 [1981], S. 98-102). 
auf und die Aufgabe des Detektivs (wie des Zeitungslesers) besteht gerade darin, diese Kennzeichnungen zu finden und aufzulösen, also z.B.: "X hat eine Uhr des Opfers gestohlen«, "X war zur Tatzeit am Tatort«, "X war in der Lage, das Verbrechen auszuüben« usw. - bis nur noch eine Person übrig bleibt.

Jolles' Ansatz ist tatsächlich problematisch, aber sein Problem ist nicht die Konfusion von Gattung und Individuum. Die Schwäche seines Ansatzes liegt darin begründet, dass er seinen morphologischen Ansatz, also die Analyse von Gestalttypen, von der historischen Dimension abzukoppeln versucht. Er kann also gerade nicht sehen, was ein historischer Vergleich von verschiedenen poetischen Formtypen über deren sozialgeschichtliche Funktionen zeigen kann. ${ }^{49}$

An Alewyns Jolles-Kritik ist außerdem zweifelhaft, ob es Jolles in den Einfachen Formen überhaupt um eine Gattungstheorie geht oder ob er nicht vielmehr (wenn auch ohne historische Spezifikation) nach den sozialen Funktionen poetischer Formen sucht. Genau das aber wäre die Frage, die sich hier am Beispiel der Kriminalliteratur stellt. Wie fügt sich das Rätsel in dieses Problemfeld?

Um ein letztes Mal Jolles zu zitieren: "Das Eigentümliche der Form Kasus liegt nun aber darin, daß sie zwar die Frage stellt, aber die Antwort nicht geben kann, daß sie uns die Pflicht der Entscheidung auferlegt, aber die Entscheidung selbst nicht enthält«. ${ }^{50}$ Die Frage, die der Kasus stellt, ist die Frage nach dem gerechten Gesetz. Er kann diese Frage aber nicht beantworten, weil er keinen absoluten Maßstab bestimmen kann, sondern das Problem immer nur kasuistisch bearbeitet. Auch das Rätsel stellt eine Frage, aber es ist die Frage nach dem individuellen Rechtsbruch, nicht die nach der abstrakten Rechtsnorm. An die Stelle der Differenz von Legalität und Legitimität tritt die Differenz von Verbrechen und seiner Erkenntnis. Das Rätsel zielt also auf eine andere Ebene, auf den Bereich der Detektion, der Ermittlungspraxis. Nur auf dieser Ebene kann es die Frage beantworten, aber es kann sie immerhin selbst beantworten. Das Rätsel ist immer auflösbar, eben weil es die Antwort in seiner Fragestellung enthält. Wenn es in der Detektiverzählung schließlich gelöst ist, ist immerhin der Rechtsfrieden wiederhergestellt - ob damit aber auch ein gerechter (und nicht nur ein rechtmäßiger) Zustand erreicht wird, verschwindet im blinden Fleck der

49 Das schließt methodologisch allerdings die Möglichkeit ein, dass sich typologisch Späteres historisch früher finden lässt und umgekehrt (zu dieser Methodologie vgl. Christian Kirchmeier: Moral und Literatur. Eine historische Typologie, München 2013, S. 77-150).

50 André Jolles: Kleine Formen, S. 191. 
Narration. In der kriminalliterarischen Poetik des Rätsels entzieht sich die Rechtsordnung der Kritik.

Die sozialen Funktionen der poetischen Form des Rätsels könnten so auch eine Gattungsfrage beantworten, die Roger Caillois aufgeworfen hat: ${ }^{51}$ Wenn nämlich die Kriminalliteratur irgendwann nur noch eine »intellektuelle[ ] Übung für scharfe Denker « ${ }^{52}$ ist, warum hält sie dann noch am literarischen Motiv des Mordes fest, ja mehr noch: warum werden die Tötungsarten mit stetig wachsender Grausamkeit dargestellt? Die Antwort könnte lauten, dass das Rätsel als poetische Form der Detektivgeschichten eben nicht nur Denksport ist, sondern das Problem der Ermittlungspraxis thematisiert. Die Detektivgeschichten stellen das Verbrechen als einen Verstoß im Rechtssystem dar, das auch durch die Mittel ebendieses Rechtssystems zu lösen ist. Es gibt dabei keine rechtstranszendente Position mehr, von der aus das Recht als potentielles Unrechtssystem kritisiert werden könnte. Die poetische Form des Rätsels hat damit das Referenzproblem der Kriminalliteratur aufgegeben, weil sie den konkreten Normbruch isoliert und so aus dem Spannungsverhältnis zu einem abstrakten Normsystem entfernt. War der beste Kasus gewissermaßen derjenige, der normativen Unsinn besonders augenscheinlich machen konnte, ist das beste Detektivrätsel dasjenige, bei dem der Fall am kniffligsten zu lösen und die Tötungsart am spektakulärsten ist. Das Rätsel des Detektivs trägt so die Signatur einer Sozialordnung, in der die Frage nach der Gerechtigkeit des Rechts nicht mehr gestellt wird. Die Detektiverzählung ist deswegen aber nicht affirmativ, wie die Kritiker der Unterhaltungskunst glauben machen wollten, sondern vielmehr der Ausdruck genau desjenigen Problems, das diese Kritiker zu lösen versuchten: Sie ist Ausdruck einer Krise der Normkritik in der Moderne.

Gerade mit Blick auf das 20. Jahrhundert müsste diese Perspektive noch einmal umgekehrt werden. Man müsste also untersuchen, wie sich soziale Semantiken in poetischen Formen niederschlagen. Die Form des detektivischen Rätsels hat Auswirkungen über die Kriminalliteratur hinaus. Denn einerseits ist der Detektiv Stellvertreter des Lesers. ${ }^{53}$ Er trägt für ihn die Indizien zusammen, die zur Lösung des Rätsels notwendig sind. Mit Blick auf die Entwicklung von Hermeneutik und Semiotik im 20. Jahrhundert kehrt sich die Perspektive aber um: Der Leser selbst wird nun zum Stellvertreter

51 Roger Caillois: Der Kriminalroman oder: Wie sich der Verstand aus der Welt zurückzieht, um seine Spiele zu spielen, und wie darin dennoch die Probleme der Gesellschaft behandelt werden, in: Der Kriminalroman, S. 157-180.

52 Ebd., S. 160.

53 Vgl. Richard Alewyn: Anatomie des Detektivromans, S. $59 f$. 
des Detektivs, auch dann, wenn er es nicht mit Kriminalliteratur zu tun hat. Er ist der literarische Ermittler, der eine versteckte Tiefenebene unter der Textoberfläche sucht; er ist der Interpret, für den die Interpretationsverweigerung literarischer Texte eine Interpretationsprovokation ist; und er ist der Zeichendeuter, der sich selbst dann noch auf die Suche nach Sinn begibt, wenn die Texte ihr Sinnpotential leugnen. 